\title{
Inhibition of Ifosfamide-Induced Urotoxicity by Disulfiram in Mice
}

\author{
Masaaki ISHIKAWA, Yoshio TAKAYANAGI and Ken-ichi SASAKI \\ Institute of Cancer Research, Tohoku College of Pharmacy. Sendai 983, Japan
}

Accepted November 4, 1988

\begin{abstract}
The effect of disulfiram on the urotoxicity induced by ifosfamide was studied in mice. Ifosfamide administered intraperitoneally to mice caused a doserelated increase in bladder weight within $48 \mathrm{hr}$ of treatment. Disulfiram prevented ifosfamide-induced bladder damage when administered orally within $1 \mathrm{hr}$ of ifosfamide treatment. The results indicate that disulfiram is an effective protective agent against bladder damage caused by ifosfamide treatment.
\end{abstract}

Ifosfamide is one of the most widely used alkylating agents in cancer chemotherapy. Urotoxicity is a characteristic, specific side effect of alkylating agents with an oxazaphosphorine ring in the molecular structure (1). Acrolein has recently been found to be a metabolite of both ifosfamide and cyclophosphamide, and the investigators proposed that it contributes to the damage caused by the urotoxicity of these compounds $(2,3)$.

In view of the generally recognized difficulty of obtaining new drugs with increased efficacy, it is important to enhance the cytotoxicity of available antitumor agents against tumor cells by reducing toxic side effects. Protection against toxic effects could allow an increase in drug dose with potential therapeutic advantages.

The protective role that thiol compounds have against the toxic effects of alkylating agents has long been recognized (4)

Recently, the parenteral administration of certain sulfhydryl-containing compounds such as $\mathrm{N}$-acetylcysteine (5), 2-mercaptoethane sulfonate (6), dimercaptosuccinic acid (7), and disulfiram (8) have been reported to prevent hemorrhagic cystitis produced by cyclophosphamide. Many of the histological lesions caused by cyclophosphamide are similar to those observed in ifosfamideinduced hemorrhagic cystitis $(2,3)$, and disulfiram has also been reported to decrease the toxicity of several alkylating agents ( 9 , 10) and to inhibit the carcinogenicity of various $\mathrm{N}$-nitroso compounds $(9,11)$.

This study was undertaken to determine whether oral administration of disulfiram influences bladder damage induced by ifosfamide treatment.

Male ddY mice, 5 weeks old and weighing 22-24 g. obtained from the Shizuoka Animal Center (Hamamatsu, Japan) were used. They were given standard rodent pellet and water ad libitum and housed at a constant temperature and humidity environment in the university vivarium. Disulfiram was obtained from Tanabe Pharmaceutical Co., Ltd., (Osaka, Japan) in the powder form. The powder was then suspended in $0.8 \%$ Tween 80-physiological saline and sonicated for $10 \mathrm{~min}$ (Braunsonic). Ifosfamide was obtained from Shionogi Pharmaceutical Co., Ltd. (Ifomide ${ }^{\circledR}$ for injection, Osaka, Japan). In all studies, ifosfamide was administered intraperitoneally, and disulfiram was administered orally in a volume of $10 \mathrm{ml} / \mathrm{kg}$ body weight. The assay of bladder toxicity, based on the drug-induced increase of bladder weight, was essentially similar to that of Brock et al. (1). Briefly, mice were given various doses of ifosfamide, i.p., and killed by cervical dislocation $48 \mathrm{hr}$ later. The bladders were excised immediately, blotted and weighed to obtain the relative wet weight to body weight for each animal (mg of bladder weight per $100 \mathrm{~g}$ of body weight). To correlate bladder weight changes with ifosfamide -induced bladder damage, bladders were fixed in $10 \%$ neutral phosphate-buffered formalin and prepared for histological study. Determination of statistically significant differences between treatment groups was 
calculated using Student's t-test.

The effect of disulfiram on the urotoxicity of ifosfamide was studied at various dose levels of both the antitumor agent and thiol compound. Ifosfamide caused a marked increase in bladder weight measured $48 \mathrm{hr}$ after drug administration at $100 \mathrm{mg} / \mathrm{kg}$ or higher in a dose-related manner (Fig. 1). The effect of disulfiram on the ifosfamideinduced changes in bladder weight was studied at various dose levels of ifosfamide. When disulfiram was administered simultaneously with ifosfamide by the oral route. as can be seen in Fig. 1, disulfiram effectively prevented the ifosfamide-induced increase in bladder weight in mice at each dose of ifosfamide. The uroprotective effect was further substantiated by histological study of bladders removed from mice treated with ifosfamide $(200 \mathrm{mg} / \mathrm{kg}$ ), ifosfamide (200 $\mathrm{mg} / \mathrm{kg}$ ) plus disulfiram (200 mg/ $\mathrm{kg})$, or saline solution. Ifosfamide administration resulted in edema of the lamina propria and muscularis, mild inflammatory reaction, and disruption of the epithelial lining. By contrast, mice given disulfiram or disulfiram plus ifosfamide had no histological evidence of bladder damage.

Since the protective efficacy of thiol compounds on the bladder is critically dependent on the timing of administration (8), further experiments were designed to determine whether the protective effect of disulfiram is dependent on administration schedule or not. When given simultaneously with ifosfamide, disulfiram prevented bladder weight increase at doses lower than $200 \mathrm{mg} /$ kg (Fig. 1). In the subsequent studies, therefore, $200 \mathrm{mg} / \mathrm{kg}$ of disulfiram was used. To determine the most effective schedule for the inhibition of ifosfamide-induced increase in bladder weight, disulfiram $(200 \mathrm{mg} / \mathrm{kg}$ ) was administered at various times before or after ifosfamide. These results are shown in Fig. 2. An oral administration of disulfiram between $60 \mathrm{~min}$ before and $60 \mathrm{~min}$ after injection of ifosfamide was effective. The protective efficacy of disulfiram was diminished in other periods of time. When administered $120 \mathrm{~min}$ before or 120 min after injection of ifosfamide, disulfiram did not protect against the increase of bladder weight.

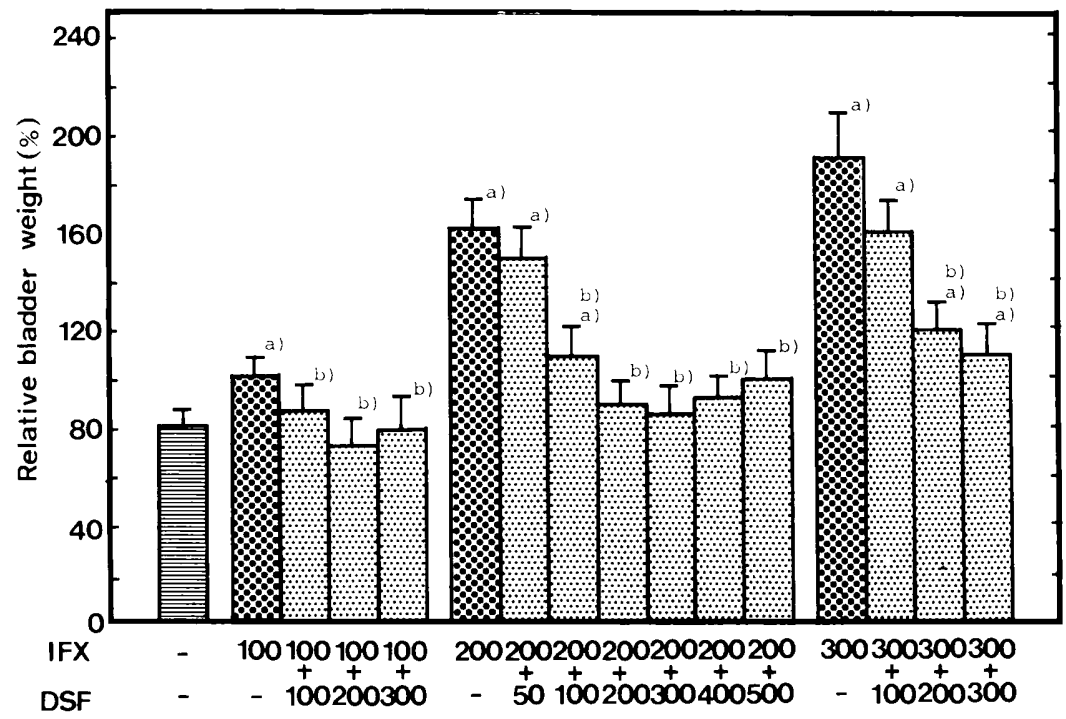

Fig. 1. Protection against ifosfamide-induced bladder toxicity by disulfiram in mice. Mice were given various doses of ifosfamide (IFX, i.p.) simultaneously with various doses of disulfiram (DSF, p.o.), and $48 \mathrm{hr}$ later, bladder weights were measured. Bladder weights were normalized to body weight (mg bladder weight per $100 \mathrm{~g}$ of body weight). Each value is presented as the mean ( \pm S.E.) of 8-10 animals. a) values significantly different from the control $(P<0.05)$. b) significantly different from ifosfamide alone $(P<0.05)$. 


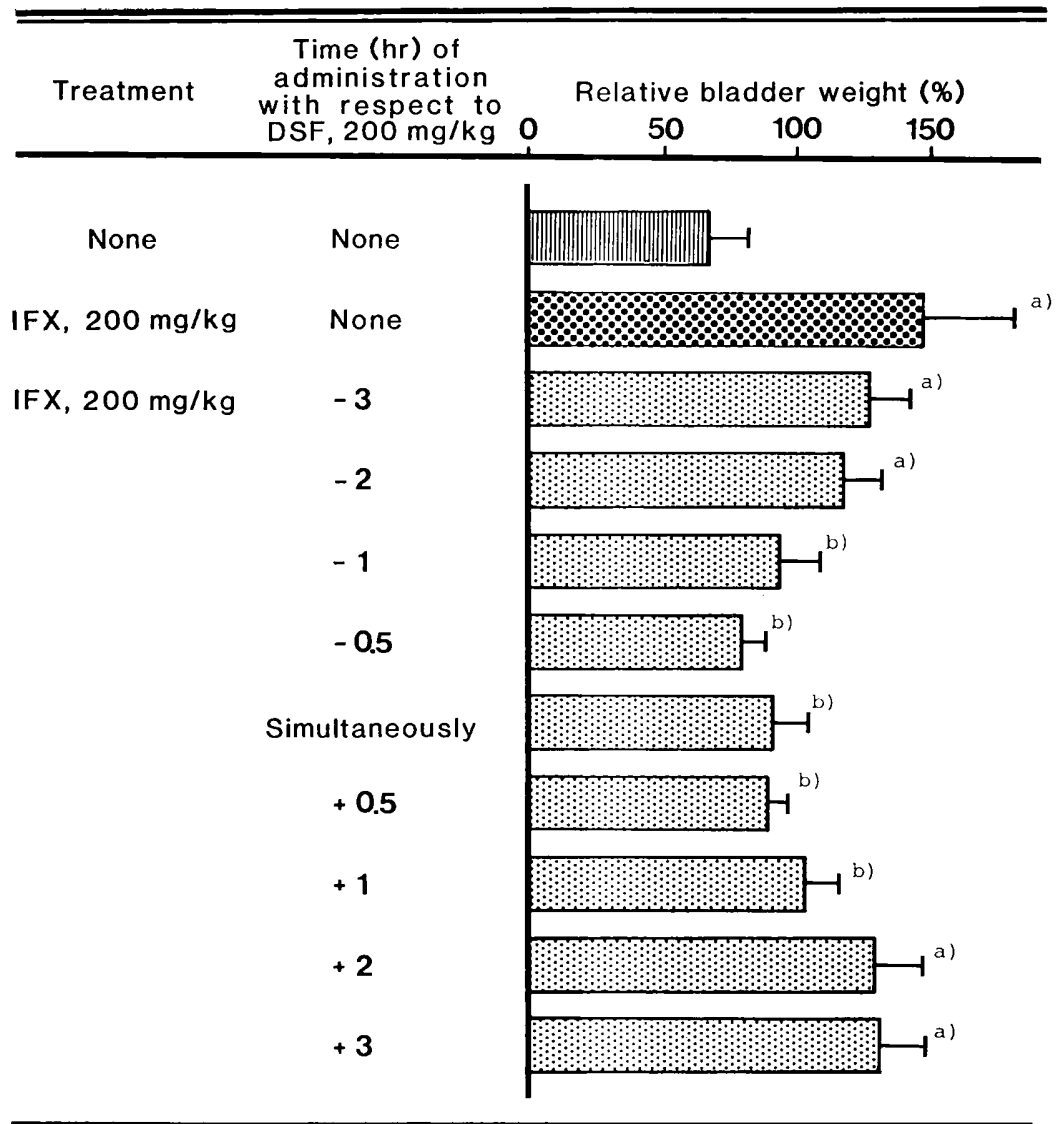

Fig. 2. Influence of treatment schedule on the protective effect of disulfiram in ifosfamide-induced bladder toxicity in mice. Mice were given ifosfamide $(200 \mathrm{mg} / \mathrm{kg}$. i.p.) and disulfiram $(200 \mathrm{mg} / \mathrm{kg}$. p.o.) at a selected time with respect to ifosfamide. Bladder weights were measured $48 \mathrm{hr}$ after ifosfamide administration. Values are the means ( \pm S.E.) for 8-10 animals. a) values significantly different from the control $(P<0.05)$. b) values significantly different from ifosfamide alone $(P<0.05)$.

Unlike most other alkylating agents, ifosfamide requires metabolic activation to attain significant cytotoxic and alkylating activity. Indeed, the metabolic products of ifosfamide, including acrolein which is characterized by weak cytotoxic properties, and chloroacetaldehyde have been implicated in this characteristic toxic effect $(1,3)$, which is a limiting factor in the therapeutic use of the drug, particularly in high-dose chemotherapy regimens.

Because of this dose-limiting toxicity of ifosfamide and related compounds, a great deal of effort has been expended in an attempt to develop a drug which is as effective as ifosfamide and lacks urotoxic potential.
Appropriate sulfhydryl-containing compounds have been reported to provide effective protection against cyclophosphamideinduced urotoxic effects, probably through deactivation of toxic metabolites $(1,5)$. In the mechanism of the protective action of these thiol compounds, a central role of endogenous glutathione has been generally recognized (12). In particular, a preferential interaction of the endogenous glutathione with the electrophilic metabolite, acrolein, has been proposed as a mechanism for protection against some toxic effects of cyclophosphamide (12). Our results, which show that simultaneously administered disulfiram is effective in preventing ifosfamide-induced 
urinary bladder changes in mice are consistent with the suggestion that endogenous glutathione has an important role in the toxicity of this alkylating agent, presumably via alkylation of reactive metabolites. Like other thiol compounds, the nucleophilic structure of glutathione would enable it to form an adduct with electrophilic drug metabolites (13). Thus, the uroprotective potential of disulfiram is not surprising. Further, unlike the other uroprotective agents, disulfiram demonstrated uroprotection when administered by the oral route. Since disulfiram is currently in clinical use and has few undesirable effects, its use in patients at high risk for ifosfamide-induced bladder injury would warrant investigation.

\section{References}

1 Brock, N., Pohl, J. and Stekar, J.: Studies on the urotoxicity of oxazaphosphorine aikylating compounds. Eur. J. Cancer 17, 595-607 (1981)

2 Alarcon, R.A. and Meienhofer, J.: Formation of the cytotoxic aldehyde acrolein during in vitro degradation of cyclophosphamide. Nature New Biol. 233, 250-252 (1971)

3 Connor, T.A.: Protection against the toxicity of alkylating agents by thiols: The mechanism of protection and its relevance to cancer chemotherapy. Eur. J. Cancer 2, 293-305 (1966)

4 Brock, N., Poho, J. and Stekar, J.: Studies on the urotoxicity of oxazaphosphorine cytostatics and its prevention. I. Experimental studies on the urotoxicity of alkylating compounds. Eur. J. Cancer 17, 595-608 (1981)
5 Tolley, D.A.: The effect of $\mathrm{N}$-acetylcysteine on cyclophosphamide cystitis. Br. J. Urol. 49, 659661 (1977)

6 Kedar, A., Simpson, C.L., Williams, P., Moore, R., Tritsch, G. and Murphy, G.P.: The prevention of cyclophosphamide induced bladder swelling in the rat by i.v. administration of sodium-2mercaptoethane sulfonate. Res. Commun. Chem. Pathol. Pharmacol. 29, 339-348 (1980)

7 Cox, P.J. and Abel, G.: Cyclophosphamide cystitis studies aimed at its minimization. Biochem. Pharmacol. 28, 3499-3502 (1979)

8 Hacker, M.P., Newman, R.A. and Ershler, W.B.: Prevention of cyclophosphamide induced cystitis by disulfiram. Fed. Proc. 41, 245 (1981)

9 Habs, H. and Habs, M.: Effect of pretreatment with disulfiram on the toxicity and antitumor activity of 1-(2-hydroxyethyl)-3-(2-chloroethyl)-3-nitrosourea in Sprague Dawley rats. Cancer Lett. 13, 63-69 (1981)

10 Borch, R.F. and Pleasants, M.E.: Inhibition of cisplatinum nephrotoxicity by diethyldithiocarbamate rescue in a rat model. Proc. Natl. Acad. Sci. U.S.A. 76 6611-6614 (1979)

11 Irving, C.C., Tice, A.J. and Murphy, W.M.: Inhibition of N-n-butyl-N-(4-hydroxybutyl)nitrosamine-induced urinary bladder cancer in rats by administration of disulfiram in the diet. Cancer Res. 39, 3040-3043 (1979)

12 Gurtoo, H.L., Hipkens, J.H. and Sharma, S.D.: Role of glutathione in the metabolism-dependent toxicity and chemotherapy of cyclophosphamide. Cancer Res. 41, 3485-3591 (1981)

13 Meister, A. and Anderson, M.E.: Glutathione. Rev. Biochem. 52, 711-760 (1983) 